$63^{\text {ème }}$ Congrès de la SFCO, 03006 (2015)

DOI: $10.1051 /$ sfco/20156303006

(C) Owned by the authors, published by EDP Sciences, 2015

\title{
POSTER
}

\section{Tumeur odontogénique kystique calcifiée à propos d'une observation clinique}

\author{
Benaouf S, Tabeti-Bentahar CF, Bouzouina F \\ Service de Pathologie et Chirurgie Buccales CHU Oran, Algérie
}

Introduction : Le kyste odontogénique calcifié (KOC) a été décrit pour la première fois par Gorlin en 1962 [Gorlin et al. 1962]. En raison de sa complexité histologique, l’OMS l'a classé en 1992 parmi les néoplasmes. En 2005, l'OMS le désigne sous le terme de tumeur odontogénique kystique calcifiée (TOKC).

Observation : Le cas rapporté est celui d'une jeune fille de 18 ans qui consulte pour une déformation osseuse symphysaire. A la radiographie panoramique une image mixte est retrouvée avec présence d'une canine (23) refoulée à la périphérie. La patiente a été opérée sous anesthésie loco-régionale et la dent a été laissée à demeure. En macroscopie, la formation était hétérogène par la présence d'un contenu solide et des structures minéralisées. L'examen histologique a conclu en faveur d'une TOKC. Six mois après, un début de réossification est constaté en périphérie et quatre années plus tard, la malade revient pour l'extraction de la 23 ayant fait son éruption en ectopie. A la radiographie, une réossification avec une cictrisation complète du site opératoire est constatée.

Discussion : les travaux de Praetorius et Ledesma-Montes ont permis d'aboutir à la classification des tumeurs odontogéniques à cellules fantômes adoptée par l'OMS en 2005 [Ledesma-Montes et al. 2008]. Celle-ci distingue entre la TOKC considérée comme la variété kystique du KOC et la tumeur dentinogénique à cellules fantômes (TDCF) qui est considérée comme sa variété solide. Leur homologue malin est aussi individualisé : il s'agit du carcinome odontogène à cellules fantômes. Pour chaque variété des sous types sont décrits dont 04 pour la TOKC [Ledesma-Montes et al. 2008]. La TOCK présente une double localisation intra-osseuse et périphérique. Elle représente $2 \%$ de l'ensemble des tumeurs odontogéniques [Knezević et al. 2004]. L'âge d'apparition se situe entre 05 et 92 ans avec un pic à la deuxième décennie [Reyes et al. 2007]. L'image radiologique de la TOKC correspond à une radiotransparence à limites bien définies avec présence possible de matériel minéralisé et de structures dentaires. Histologiquement, la présence de cellules fantômes dues à une kératinisation aberrante n'est pas pathognomonique [Praetorius et al. 1981]. La formation de matériel ostéoïde ou dentinoïde serait due à une réponse inflammatoire des tissus gingivaux aux amas de cellules fantômes [Gorlin et al. 1962]. Par ailleurs, la TOKC se caractérise par la présence de structures épithéliales pluristratifiées et des cellules pré-améloblastiques rappelant l'organe de l'émail [Kler et al. 2009]. En immunohistochimie, la TOKC exprime la cytokératine K10/13 et K14 [Crivellini 2009]. Sur le plan évolutif, cette tumeur se distingue par sa bénignité constante cependant des cas de transformation maligne et d'améloblastome ex- TOKC sont décrits [Ide et al. 2005].

This is an Open Access article distributed under the terms of the Creative Commons Attribution License 4.0, which permits unrestricted use, distribution, and reproduction in any medium, provided the original work is properly cited. 
Conclusion : les tumeurs odontogéniques à cellules fantômes sont des entités différentes en théorie. En réalité, l'absence de critères différentiels suffisamment clairs et spécifiques et l'abondance de dénominations et classifications rendent le diagnostic difficile et expliquent les nombreuses controverses et confusions.

Nom et adresse du conférencier

Souad BENAOUF

Faculté de médecine d'Oran

114, coopérative Marhaba ES Senia Oran

31100 Oran (Algérie)

s.benaouf@yahoo.fr 